\title{
Rotor speed, load torque and parameters estimations of a permanent magnet synchronous motor using extended observer forms
}

\author{
Ramdane Tami ${ }^{1, *}$, Driss Boutat ${ }^{2}$, Gang Zheng ${ }^{3}$, Frédéric Kratz ${ }^{2}$, Rachid El Gouri ${ }^{4}$ \\ ${ }^{1}$ Université de Caen Basse Normandie, UMR 6072 Laboratoire GREYC, Boulevard Maréchal \\ Juin, 14032 Caen, France \\ ${ }^{2}$ INSA Centre Val de Loire, Université d'Orléans, PRISME EA 4229, 18022 Bourges, France \\ ${ }^{3}$ INRIA Lille-Nord Europe, 40 Avenue Halley 59650, France \\ ${ }^{4}$ Laboratoire Ingénierie des Systèmes Electriques et des Télécommunications de 1'ENSA de \\ Kénitra, Morocco \\ *ramdane.tami@unicaen.fr
}

\begin{abstract}
This paper presents a new approach to estimate states and parameters of the permanent magnet synchronous motor (PMSM) in the presence of unknown load torque disturbance. Indeed, it highlights an auxiliary dynamics that is added to the PMSM model. Thereby, it supplies a change of coordinates that transforms the extended model (PMSM model together with the auxiliary dynamics) into the so-called extended nonlinear observer forms. It turns out that the obtained form provides two observer normal forms. One of them supports the well-know high gain observer which is used to estimate the speed and the unknown load torque. The second one which is affine in the parameters enables us to estimate simultaneously the states and motor parameters by means of an adaptive observer. Performance of the proposed method is illustrated by the simulations results.
\end{abstract}

\section{Introduction}

Permanent magnet synchronous motor (PMSM) is increasingly used in several industrial applications, like renewable energy and Maglev (derived from magnetic levitation) due to some important features as stability, high power density and large torque to current ratio (see [56], [28]). However, the control of the PMSM is a complex task due to the presence of nonlinearities in its model, load torque perturbations and wear occurring in mechanical sensors. This leads to, inter alia, to the development of sensorless control method which can be represented by three main approaches: the first one is based on back-emf technique (see [37], [35], [17]) whose advantage is the simplicity of implementation. However, it should be avoided in low speed region because on one hand, the velocity and the flux estimation are sensitive to the stator resistance and on the other hand, the noise amplitude is higher than back-emf. The second one is the model reference adaptive system (MRAS) method in which the performance depends on the accuracy of the used model (see [29], [41]). Finally, the third one, that will be considered in 
this paper, is based on the observer design as in ([54], [6]).

A high performance of a control requires measurement and identification of unknown parameters. Therefore, an accurate estimation of unavailable state variables is needed. In order to reach this goal, numerous observer strategies have been developed to estimate states and parameters of PMSM. Among these strategies, for example, we find exact linearization method (see [13], [55]), adaptive observer [3], sliding mode observer (see [18], [24], [40]) and extended Kalman filter (see [4], [21]).

Another interesting approach can be found in [19] where an adaptive interconnected observer has been proposed. Indeed, the dynamical system of PMSM was considered as an interaction between two sub dynamical systems: one contains the dynamics of current $i_{q}$, the stator resistance $R_{s}$ and the second one is governed by the dynamics of current $i_{d}$, angular speed $\Omega$ and load torque $T_{l}$.

In the literature, several works dealt with observer design for PMSM on the one hand, to avoid the use of tachometer which is expensive, on the other hand to ensure redundancy of information. However, few solutions have been proposed to estimate simultaneously the states, disturbances and all parameters which are required for example in diagnosis and fault-tolerant control.

When a nonlinear dynamical system contain unavailable states for measurements, then it becomes a difficult task to directly apply the standard estimation methods, such as the Luenberger's observer or Kalman's filter. To overcome this problem, Krener and Isidori [25] introduced the so-called linearization of a nonlinear dynamical system by means of a change of coordinates and output injection. This enables us to use the well-known Luenberger's observer for a class of nonlinear dynamical systems. Since, this concept has been used to the multiple outputs case in ([26] and [48],[10],[53]). Afterwards, several variations have been developed in ([23], [39], [43], [20], [33], [8], [31] and [30],[51],[50],[5]). Another interesting form is the so-called output depending observer normal form introduced by [42], and then improved in [52] and [47]. Recently, the so-called extended observer normal form was proposed in ([22], [36], [2], [7] and [9]). The idea behind this normal form is based on adding an auxiliary dynamics into the original system such that the augmented system fulfills conditions which guarantee the transformation of the nonlinear system into an extended observer normal form. Even now, the process of transforming nonlinear dynamical systems into nonlinear observer forms for a wide class of nonlinear systems keep going, as the extended output depending observer normal form is proposed in [44].

This paper presents a new approach to design nonlinear observer for PMSM by adding an auxiliary dynamics to the PMSM model such that the augmented dynamical system admits a change of coordinates that brings it into the so-called extended nonlinear observer form. This form enables us to effectively solve the problem of simultaneous estimation of PMSM states and parameters [32]. The present work improves our previous work in [45]. In fact, [45] is focused only on reaching the observer normal forms for PMSM model. Hence simulations were rather did for the mathematical model without worrying on the physical model. Therefore it contains many gaps and defects. The present study is more complete because it addresses the problem of estimation of rotor position, rotor speed, load torque, currents and the parameters. The quality of the estimation, despite the presence of a varying load torque, shows the robustness of this method to the disturbances. 
The rest of this paper is organized as follows. Section 2 presents the dynamical model of PMSM and recalls the extended nonlinear observer form structure. Section 3 states an auxiliary dynamics and a change of coordinates that transforms the extended PMSM model into an extended nonlinear observer form. Furthermore, two cases will be considered. The first one assumes the knowledge of the parameters of PMSM and the second one does not. Section 4 provides simulation results that highlight the efficiency of the proposed approach.

\section{Mathematical model of PMSM and Problem statement}

In order to obtain a dynamical model that makes simpler the estimation and control procedures, the electrical and mechanical dynamics of PMSM are expressed in the direct and quadrature axis reference frame by transforming the original three-phase on two fictitious phase winding. The direct phase winding is parallel to the rotor magnet flux, and the quadrature phase winding is orthogonal to the direct phase winding. Based on states observer, the sensorless control system is shown in Figure 1. To avoid the flux weakening, a zero electric current is required in the direct phase, then the generated electromagnetic torque is given as product of constant permanent flux and electric current in direct axis. In this present study, we consider the cases of non-saliency rotor $\left(L_{d}=L_{q}=L_{s}\right)$. The dynamical model of PMSM in $(d-q)$ reference frame is governed by the following set of differential equations (see [12], [38]).

$$
\begin{aligned}
\frac{d \theta}{d t} & =\Omega \\
\frac{d \Omega}{d t} & =-\frac{f_{v}}{J} \Omega+\frac{p}{J} \phi_{f} i_{q}-\frac{1}{J} T_{l} \\
\frac{d i_{d}}{d t} & =-\frac{R_{s}}{L_{s}} i_{d}+p \Omega i_{q}+\frac{1}{L_{s}} u_{d} \\
\frac{d i_{q}}{d t} & =-\frac{R_{s}}{L_{s}} i_{q}-p \Omega i_{d}-p \frac{1}{L_{s}} \phi_{f} \Omega+\frac{1}{L_{s}} u_{q}
\end{aligned}
$$

where $\left[\theta, \Omega, i_{d}, i_{q}\right]^{T}$ represents the state vector of the rotor angular position, the rotor mechanical speed and the output currents respectively. $u_{d}$ and $u_{q}$ are the $d$-axis and $q$-axis stator voltages, respectively. $T_{l}$ is the load torque considered as an unknown exogenous input, $R_{s}$ is the winding stator resistance, $L_{s}$ is the stator winding inductance, $\phi_{f}$ is the permanent magnet flux, $p$ is the number of the pair poles, $J$ is the rotor moment of inertia, and $f_{v}$ is the viscous friction coefficient.

Within this work it is assumed that the available measurements are the currents in the $(d-q)$ reference frame. Thus, the outputs of the above dynamical system are as follows

$$
\begin{aligned}
& y_{1}=h_{1}=i_{d} \\
& y_{2}=h_{2}=i_{q}
\end{aligned}
$$

Motivated by the design of robust controllers and faults diagnosis strategies for PMSM applications, as it has been widely mentioned above in the introduction, many works had been conducted to estimate states and parameters of PMSM. Hereafter, we will present a new approach which enables us to: 
- estimate the rotor mechanical speed $\Omega$ and the load torque $T_{l}$ when all motor parameters are known;

- estimate the parameters $R_{s}, L_{s}$ and $\phi_{f}$;

- consequently, estimate the rotor angular position from the estimation of rotor mechanical speed.

In order to design an observer for PMSM model, it is necessary to study its observability property. This also enables us to effectively manage the loss of observability when it occurs. The rank observability conditions (see for example [25]) for system (2)-(4) together with the measurable outputs $i_{d}$ and $i_{q}$ is the rank of the following observability matrix

$$
\mathcal{O}=\left[\begin{array}{c}
d h_{1} \\
d h_{2} \\
d L_{f} h_{1} \\
d L_{f} h_{2}
\end{array}\right]=\left[\begin{array}{ccc}
0 & 1 & 0 \\
0 & 0 & 1 \\
p i_{q} & -\frac{R_{s}}{L_{s}} & p \Omega \\
-\left(\frac{p}{L_{s}} \phi_{f}+p i_{d}\right) & -p \Omega & -\frac{R_{s}}{L_{s}}
\end{array}\right]
$$

A straightforward calculation shows that the rank of the above matrix is

$$
\operatorname{rank}(\mathcal{O})=3
$$

if and only if $i_{d} \neq-\frac{\phi_{f}}{L_{s}}$ or $i_{q} \neq 0$.

As stated in the introduction, we will use the well-known estimation approach based on the extended nonlinear observer normal form. In order to do so, let us recall the basic idea and some properties of this concept. Consider the following nonlinear system

$$
\left\{\begin{array}{l}
\dot{x}=f(x) \\
y=h(x)
\end{array}\right.
$$

where $x \in U \subseteq \mathbb{R}^{n}$ represents the state and $y \in \mathbb{R}$ denotes the outputs. We assume that the vector field $f$ and the output function $h$ are sufficiently smooth. It is also assumed that the pair $(h, f)$ satisfies the observability rank condition. Thus, the 1 -forms $\theta_{i}=d L_{f}^{i-1} h$, for $1 \leq i \leq n$, are linearly independent where $L_{f}^{i-1} h$ is the $(i-1)^{t h}$ Lie derivative of the output $h$ along the vector field $f$ and $d$ the usual differential.

When system (7) cannot be transformed into an observer normal form by means of the socalled linearization by output injection [25], then we can overcome this limitation by using the immersion concept (dynamics extension). The idea is to seek for an auxiliary dynamics $\dot{w}=\eta(y, w)$ with $w \in \mathbb{R}$ so that the following extended dynamical system

$$
\begin{aligned}
\dot{x} & =f(x) \\
\dot{w} & =\eta(y, w) \\
y & =h(x)
\end{aligned}
$$

might be transformed by means of a change of coordinates $\left(\xi^{T}, w\right)^{T}=\phi(x, w)$ to the following extended observer normal form

$$
\begin{aligned}
\dot{\xi} & =A(y, w) \xi+B(y, w) \\
\dot{w} & =B_{n+1}(y, w) \\
y & =C \xi
\end{aligned}
$$


where $w \in \mathbb{R}$ is an auxiliary variable considered as an extra output, $C=[0, \ldots, 0,1]$, and

$$
A(y, w)=\left(\begin{array}{cccc}
0 & & \cdots & 0 \\
\alpha_{2}(y, w) & \ddots & & \vdots \\
0 & \ddots & \ddots & \\
\vdots & & & \\
0 & \cdots 0 & \alpha_{n}(y, w) & 0
\end{array}\right)
$$

where $\alpha_{i}(y, w) \neq 0$ for $2 \leq i \leq n$ are functions depending only on the output $y$ and the extra output $w$.

This extended nonlinear observer normal form supports the high gain observer to estimate the state of nonlinear dynamical system [11]. Moreover, in the case of PMSM model we will see that $B($.$) is affine on some parameters, therefore the adaptive observer can be used to estimate$ simultaneously the states and the parameters (see [32], [49]).

\section{Change of coordinates and Extended dynamics}

This section provides an auxiliary dynamics together with a change of coordinates that transforms the extended PMSM model (2)-(4) into an extended nonlinear observer normal form.

Let us consider the equations (2)-(4) together with an auxiliary dynamics presented as follows

$$
\begin{aligned}
\dot{x}_{1} & =-\frac{f_{v}}{J} x_{1}+\frac{p}{J} \phi_{f} x_{3}-\frac{1}{J} T_{l} \\
\dot{x}_{2} & =-\frac{R_{s}}{L_{s}} x_{2}+p x_{1} x_{3}+\frac{1}{L_{s}} u_{d} \\
\dot{x}_{3} & =-\frac{R_{s}}{L_{s}} x_{3}-p x_{1} x_{2}-p \frac{1}{L_{s}} \phi_{f} x_{1}+\frac{1}{L_{s}} u_{q} \\
\dot{w} & =\eta(y, w) \\
y_{1} & =x_{2} \\
y_{2} & =x_{3}
\end{aligned}
$$

where $\left[x_{1}, x_{2}, x_{3}\right]=\left[\Omega, i_{d}, i_{q}\right], y=\left[y_{1}, y_{2}\right]^{T}$ and $w$ is an auxiliary variable considered as an extra output.

Then the following result provides the sought auxiliary dynamics $\eta(y, w)$ and the change of coordinates.

Theorem 1. For any function $\kappa(w) \neq 0$, consider the following auxiliary dynamics

$$
\dot{w}=-\frac{f_{v}}{J} \kappa(w)
$$


Then, the following change of coordinates

$$
\begin{aligned}
& z_{1}=x_{1} e^{-\int_{0}^{w} \frac{1}{\kappa(s)} d s} \\
& z_{2}=x_{2} \\
& z_{3}=x_{3}
\end{aligned}
$$

transforms the nonlinear dynamical system (15)-(17) into following form

$$
\begin{aligned}
& \dot{z}_{1}=\alpha_{1}(w) T_{l}+\beta_{1}(y, w) \\
& \dot{z}_{2}=\alpha_{2}(y, w) z_{1}+\beta_{2}(y, w)+\frac{1}{L_{s}} u_{d} \\
& \dot{z}_{3}=\alpha_{3}(y, w) z_{2}+\beta_{3}(y, w)+\frac{1}{L_{s}} u_{q}
\end{aligned}
$$

where

$$
\begin{aligned}
& \alpha_{1}(y, w)=-\frac{1}{J} e^{-\int_{0}^{w} \frac{1}{\kappa(s)} d s}, \alpha_{2}(y, w)=p y_{2} e^{\int_{0}^{w} \frac{1}{\kappa(s)} d s}, \alpha_{3}(y, w)=-p\left(y_{1}+\frac{\phi_{f}}{L_{s}}\right) e^{\int_{0}^{w} \frac{1}{\kappa(s)} d s}, \\
& \beta_{2}(y, w)=-\frac{R_{s}}{L_{s}} y_{1}, \beta_{1}(y, w)=\frac{p}{J} \phi_{f} y_{2} e^{-\int_{0}^{w} \frac{1}{\kappa(s)} d s}, \text { and } \beta_{3}(y, w)=-\frac{R_{s}}{L_{s}} y_{2}
\end{aligned}
$$

Remark 1. - Function $\kappa(w) \neq 0$ is a free function. It has to be chosen in order to ensure the boundedness the auxiliary dynamics. For our goal, more details on this function are given in simulation section.

- As it can be seen, the PMSM outputs considered here are continuous-time measurements. In practice, with high frequency sampling device, the measurement can be considered as continuous. Even with a very low sampling sensor (rarely), thanks to our observer normal form, it is easy to adapt the observer proposed in [16] to work with sampled-outputs.

Proof. The time derivative of the state variable $z_{1}$ given in equation (20) leads to

$$
\dot{z}_{1}=-\frac{1}{J} e^{-\int_{0}^{w} \frac{1}{\kappa(s)} d s} T_{l}+\frac{p}{J} \phi_{f} y_{2} e^{-\int_{0}^{w} \frac{1}{\kappa(s)} d s} .
$$

by setting

$$
\begin{aligned}
\alpha_{1}(y, w) & =-\frac{1}{J} e^{-\int_{0}^{w} \frac{1}{\kappa(s)} d s} \\
\beta_{1}(y, w) & =\frac{p}{J} \phi_{f} y_{2} e^{-\int_{0}^{w} \frac{1}{\kappa(s)} d s}
\end{aligned}
$$

hence equation (26) can be rewritten in the following form:

$$
\dot{z}_{1}=\alpha_{1}(y, w) T_{l}+\beta_{1}(y, w)
$$

Now by replacing the variables $x_{2}=z_{2}$ and $x_{3}=z_{3}$ in equations (16) and (17), we obtain 


$$
\begin{aligned}
\dot{z}_{2} & =p y_{2} e^{\int_{0}^{w} \frac{1}{\kappa(s)} d s} z_{1}-\frac{R_{s}}{L_{s}} y_{1}+\frac{1}{L_{s}} u_{d} \\
& =\alpha_{2}(y, w) z_{1}+\beta_{2}(y, w)+\frac{1}{L_{s}} u_{d}
\end{aligned}
$$

and

$$
\begin{aligned}
\dot{z}_{3} & =-p\left(y_{1}+\frac{\phi_{f}}{L_{s}}\right) e^{\int_{0}^{w} \frac{1}{\kappa(s)} d s} z_{2}-\frac{R_{s}}{L_{s}} y_{2}+\frac{1}{L_{s}} u_{q} \\
& =\alpha_{3}(y, w) z_{2}+\beta_{3}(y, w) .
\end{aligned}
$$

Putting together equations (26), (28) and (29) to meet the stated nonlinear extended normal form of the above theorem.

Now, to design an observer for PMSM relying on the extended nonlinear observer normal form obtained in Theorem 1, two case studies are to be considered:

- estimation of rotor mechanical speed $z_{1}=\omega$ and the load torque $T_{l}$ by using equations (23) and (25) where all parameters of the system are assumed known,

- estimation of $z_{1}=\omega$, the load torque $T_{l}$, and the parameters $R_{s}, L_{s}$ and $\phi_{f}$ by involving dynamics (23) and (24).

Remark 2. As proposed in [19], we will use the following observer to estimate the rotor position

$$
\frac{d \widehat{\theta}}{d t}=\widehat{\Omega}+L_{\theta}\left(i_{q}-\widehat{i}_{q}\right)
$$

where $L_{\theta}$ is a positive constant design parameter.

\subsection{Estimation of motor speed and the load torque with the known parameters}

In this subsection, it is assumed that all parameters of PMSM are known and the load torque $T_{l}$ is a piece-wise constant function $\left(\dot{T}_{l}=0\right)$. Now, in order to estimate the rotors speed and the load torque, we consider equation $\dot{T}_{l}=0$ together with dynamics $\dot{z}_{1}$ and $\dot{z}_{3}$ obtained from the extended nonlinear observer form given in Theorem 1 . Thus, we consider the following nonlinear extended observer normal form:

$$
\begin{aligned}
& {\left[\begin{array}{c}
\dot{T}_{l} \\
\dot{z}_{1} \\
\dot{z}_{3}
\end{array}\right]=\left[\begin{array}{ccc}
0 & 0 & 0 \\
\alpha_{1}(y, w) & 0 & 0 \\
0 & \alpha_{3}(y, w) & 0
\end{array}\right]\left[\begin{array}{c}
T_{l} \\
z_{1} \\
z_{3}
\end{array}\right]+\left(\begin{array}{c}
0 \\
\beta_{1}(y, w) \\
\beta_{3}(y, w, u)
\end{array}\right)} \\
& \dot{w}=-\frac{f_{v}}{J} \kappa(w)
\end{aligned}
$$

where $\alpha_{1}(y, w)=-\frac{e^{-\int_{0}^{w} \frac{1}{\kappa(s)} d s}}{J}, \alpha_{3}(y, w)=-p\left(y_{1}+\frac{\phi_{f}}{L_{s}}\right) e^{\int_{0}^{w} \frac{1}{\kappa(s)} d s}, \beta_{1}(y, w)=\frac{p}{J} \phi_{f} y_{2} e^{-\int_{0}^{w} \frac{1}{\kappa(s)} d s}$ and $\beta_{3}(y, w, u)=-\frac{R_{s}}{L_{s}} y_{2}+\frac{1}{L_{s}} u_{q}$

In order to design an observer for the above dynamical system, let us set: 


$$
A(y, w)=\left[\begin{array}{ccc}
0 & 0 & 0 \\
\alpha_{1}(y, w) & 0 & 0 \\
0 & \alpha_{3}(y, w) & 0
\end{array}\right] \text { and } B(y, u)=\left(\begin{array}{c}
0 \\
\beta_{1}(y, w) \\
\beta_{3}(y, w, u)
\end{array}\right) .
$$

Now, as in [11], let us consider this new dynamical system:

$$
\begin{aligned}
& \dot{\hat{\xi}}=A(y, w) \hat{\xi}+B(y, w, u)-\Gamma^{-1}(y) R_{\rho}^{-1} C^{T}(C \hat{\xi}-y) \\
& 0=\rho R_{\rho}+G^{T} R_{\rho}+R_{\rho} G-C^{T} C
\end{aligned}
$$

where

$$
G=\left(\begin{array}{ccc}
0 & 0 & 0 \\
1 & 0 & 0 \\
0 & 1 & 0
\end{array}\right), \Gamma(y, w)=\left(\begin{array}{ccc}
\alpha_{1} \alpha_{3} & 0 & 0 \\
0 & \alpha_{3} & 0 \\
0 & 0 & 1
\end{array}\right)
$$

and from the last algebraic equation we can deduce that:

$$
R_{\rho}=\left[\begin{array}{ccc}
\frac{6}{\rho^{5}} & \frac{-3}{\rho^{4}} & \frac{1}{\rho^{3}} \\
\frac{-3}{\rho^{4}} & \frac{2}{\rho^{3}} & \frac{-1}{\rho^{2}} \\
\frac{1}{\rho^{3}} & \frac{-1}{\rho^{2}} & \frac{1}{\rho}
\end{array}\right]
$$

It is worth noting that the term $-\Gamma^{-1}(y) R_{\rho}^{-1} C^{T}$ has the following simple expression:

$$
\left[-\frac{\rho^{3}}{\alpha_{1} \alpha_{3}},-3 \frac{\rho^{2}}{\alpha_{3}},-3 \rho\right]^{T}
$$

It is well-known from [11] that if the output $y$ and the extra-output $w$, functions $\alpha_{1}(y, w)$ and $\alpha_{3}(y, w)$ are bounded, then the dynamical system (31) is an observer for (26). Thus the error observation $e=\hat{\xi}-\xi$ which is governed by the following dynamics:

$$
\dot{e}=\dot{\hat{\xi}}-\dot{\xi}=\left(A(y, w)-\Gamma^{-1}(y, w) R_{\rho}^{-1} C^{T} C\right) e=\bar{A}(y, w) e
$$

where the matrix $\bar{A}(y, w)$ has the following expression $\bar{A}(y, w)=\left(\begin{array}{ccc}0 & 0 & -\frac{\rho^{3}}{\alpha_{1} \alpha_{3}} \\ \alpha_{1} & 0 & -3 \frac{\rho^{2}}{\alpha_{3}} \\ 0 & \alpha_{3} & -3 \rho\end{array}\right)$, is exponentially stable by an appropriate choice of $\rho$.

As in many works that deal with high gain observers, in our normal form we don't need to do any further assumption (as Lipschitz condition) on $B(y, w, u)$ because it depends only on measurable quantities.

\subsection{Estimation of motor speed, the load torque and identification parameters}

In this subsection, it is assumed that parameters $\phi_{f}, L_{s}$ and $R_{s}$ are unknown. Then, in order to deal with simultaneously estimation of states and parameters of dynamical system (2)-(4), we consider equation $\dot{T}_{l}=0$ together with dynamics $\dot{z}_{1}$ and $\dot{z}_{2}$ obtained from the extended nonlinear observer forms given in Theorem 1 . Thus, we consider the following extended nonlinear observer forms 


$$
\left\{\begin{array}{l}
\dot{T}_{l}=0 \\
\dot{z}_{1}=-\frac{1}{J} e^{-\int_{0}^{w} \frac{1}{\kappa(s)} d s} T_{l}+\frac{p}{J} \phi_{f} y_{2} e^{-\int_{0}^{w} \frac{1}{\kappa(s)} d s} \\
\dot{z}_{2}=p y_{2} e^{\int_{0}^{w} \frac{1}{\kappa(s)} d s} z_{1}-\frac{R_{s}}{L_{s}} y_{1}+\frac{1}{L_{s}} u_{d} \\
\dot{w}=-\frac{f_{v}}{J} \kappa(w)
\end{array}\right.
$$

It is clear that this form is of the following adaptive observer form (affine on parameters)

$$
\left\{\begin{array}{l}
\dot{z}=A(w, y, u) z+\phi(w, u, y) \delta \\
y=C z
\end{array}\right.
$$

where $z=\left[\begin{array}{lll}T_{l} & z_{1} & z_{2}\end{array}\right]^{T}, \delta=\left[\begin{array}{lll}\phi_{f} & \frac{R_{s}}{L_{s}} & \frac{1}{L_{s}}\end{array}\right]^{T}$

$$
A(w, y)=\left[\begin{array}{ccc}
0 & 0 & 0 \\
\frac{-1}{J} e^{-\int_{0}^{w} \frac{1}{\kappa(s)} d s} & 0 & 0 \\
0 & p y_{2} e^{\int_{0}^{w} \frac{1}{\kappa(s)} d s} & 0
\end{array}\right]
$$

and

$$
\phi(w, u, y)=\left[\begin{array}{ccc}
0 & 0 & 0 \\
\frac{p}{J} y_{2} e^{-\int_{0}^{w} \frac{1}{\kappa(s)} d s} & 0 & 0 \\
0 & -y_{1} & u_{d}
\end{array}\right]
$$

Now, as in [32, 49], let us consider the following system:

$$
\begin{aligned}
& \dot{\widehat{z}}=A(y, w) \widehat{z}+\phi(w, u, y) \widehat{\delta}+\left\{\Lambda S_{\delta}^{-1} \Lambda^{T} C^{T}+S_{z}^{-1} C^{T}\right\} \Sigma(y-C \widehat{z}) \\
& \dot{\widehat{\delta}}=S_{\delta}^{-1} \Lambda^{T} C^{T} \Sigma(y-C \widehat{z})
\end{aligned}
$$

where $\Lambda, S_{z}, S_{\delta}$ are respectively the solutions of the following equations:

$$
\begin{aligned}
\dot{\Lambda} & =\left\{A(y, w)-S_{z}^{-1} C^{T} C\right\} \Lambda+\phi(w, u, y) \\
\dot{S}_{z} & =-\rho_{z} S_{z}-A(y, w)^{T} S_{z}-S_{z} A(y, w)+C^{T} \Sigma C \\
\dot{S}_{\delta} & =-\rho_{\delta} S_{\delta}+\Lambda^{T} C^{T} \Sigma C \Lambda, \quad S_{z}(0), S_{\delta}(0)>0
\end{aligned}
$$

where $\rho_{z}$ and $\rho_{\theta}$ are positive constants.

Under the following persistence of excitation condition: there exist $\lambda>0$ and $\sigma>0$ such that

$$
\lambda I \leq \int_{t}^{t+T} \Lambda(\tau)^{T} C^{T} \Sigma(\tau) C \Lambda(\tau) d \tau \leq \sigma I,
$$

it has been proven in [32, 49] that dynamical system (34)- (35) is an observer for dynamical system (32). Thus, it enables us to estimate exponentially the state $z$ and the parameters $\delta$ for system (32). To validate the theoretical part of proposed approach, we will present the numerical results that reflect the good dynamics performance and fast estimation convergence to their real trajectories. 


\begin{tabular}{|c|c||c|c|}
\hline Parameter & Value & Parameter & Value \\
\hline Stator resistance $\left(R_{s}\right)$ & $2.875 \Omega$ & Number of pole pairs $(p)$ & 3 \\
\hline Stator inductance $\left(L_{s}\right)$ & $8.5 \mathrm{mH}$ & Rotor magnetic flux $\left(\phi_{f}\right)$ & $0.175 \mathrm{~Wb}$ \\
\hline Moment of inertia $(J)$ & $0.00003 \mathrm{kgm}^{2}$ & Viscous friction coefficient $\left(f_{v}\right)$ & $0.0034 \mathrm{Nm} / \mathrm{s}$ \\
\hline
\end{tabular}

Table 1 TABLE MOTOR PARAMETERS

\section{Simulation results}

The Table-I provides mechanical/electrical data for PMSM model used in simulation. The structure sensorless control system is depicted in Fig.1. The structure sensorless control system

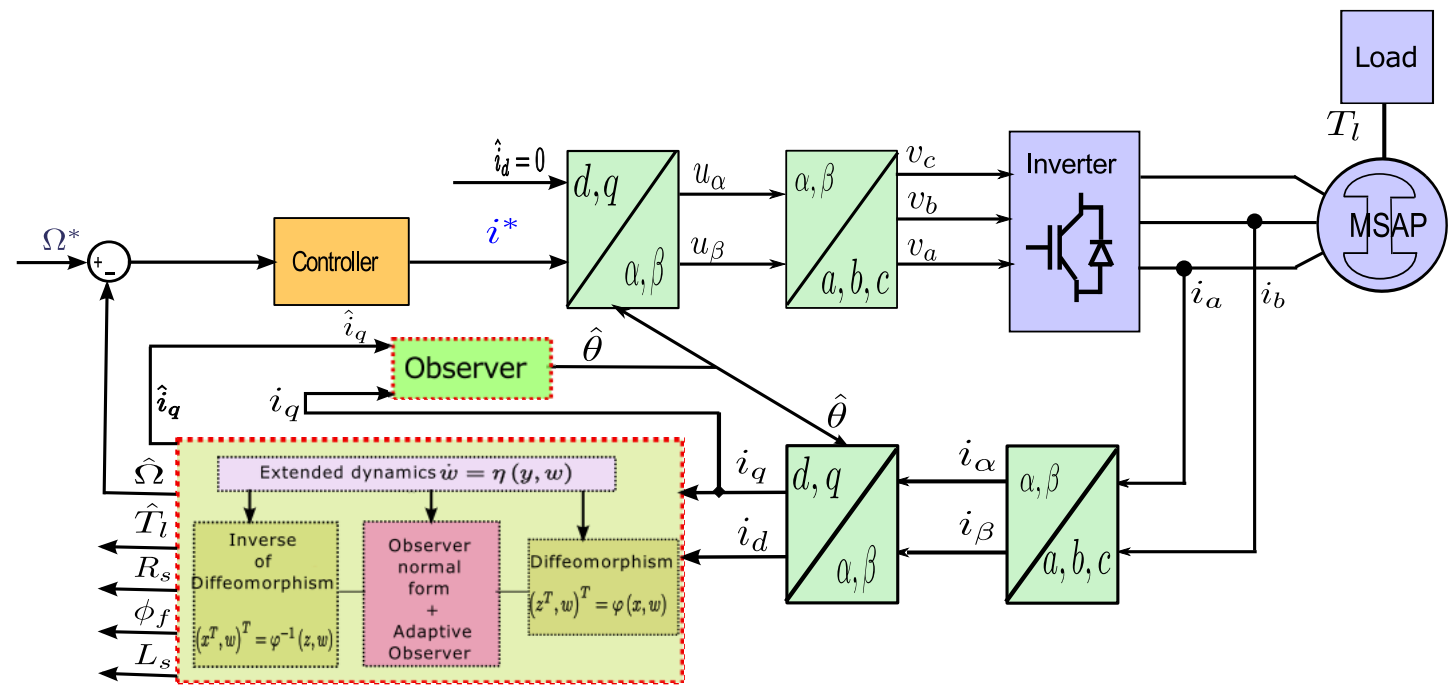

Fig. 1: Implementation of an Adaptive observer through a change of coordinates.

is depicted in Fig.1, where a zero electric current is setting in the direct phase $i_{d}^{*}$ because the flux does not need to be controlled since it is constant in the case of non-saliency rotor and in order to optimize the torque-to-current ratio. In order to ensure the boundedness of extended dynamics (19), we take $\kappa(w)=\frac{\sin ^{2} a w}{(a w)^{2}} \neq 0$ for $|w|<\frac{\pi}{a}$. The parameter $a$ is chosen such that the simulation interval where $w$ is bounded as wide as we want. In the present study, we take $a=0,0005$ to have an interval $[-5,5]$ (see Fig.2 below). In order to verify the efficiency of the proposed approaches, we use the input proposed in [15] to excite the motor under low and high transitions of rotor speed such that the observability and the PE conditions can be satisfied.

Remark 3. Fig. 1 presents the general structure of observer-based control of motor. However, this paper deals only with the observer design problem and no controller has been investigated. Different kinds of controllers have already been proposed in the literature, such as observerbased sliding mode controller [46, 19], where the stability of the closed-loop system has been studied in the theoretical and practical situations. Interested reader can refer to the references therein. 


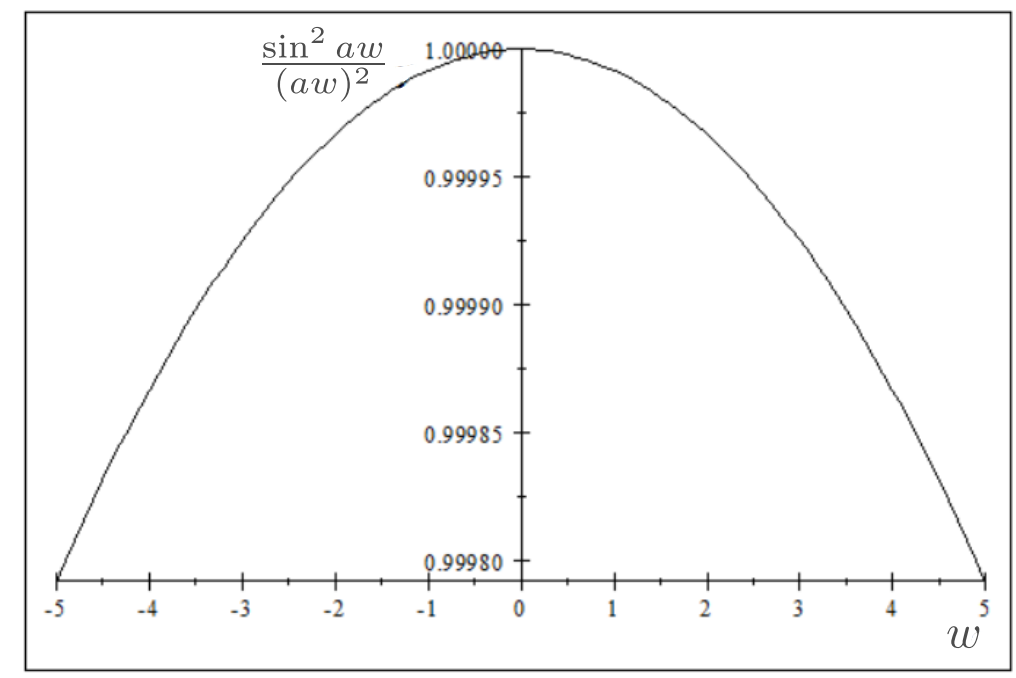

Fig. 2: Shape of $\frac{\sin ^{2} a w}{(a w)^{2}}$.

\subsection{Simulation results with high gain observer}

When the parameters are assumed known, we consider the observer normal form (30) and the high gain observer (31) with $\rho=40$. The efficiency of the proposed approach which estimates the rotor position, rotor speed and load torque is shown in Fig.3 - Fig.4.

To better highlight the role of the proposed observer normal form, we perform a comparison with sliding mode observer (SMO) [24], [40]. Although, for some classes of nonlinear systems particularly in presence of faults and uncertainties, the SMO is combined to the high gain observer to bring robustness in the estimation process. Concerning the famous chattering phenomenon, it is well known that several methods can be used to deduce it, like filter [27], sigmoid function, discrete SMO [1,34] and so on. However, those modifications provide as well some disadvantages. For example, the introduction of low-pass filter can definitely reduce the chattering, but it will slightly introduce delay. Replacing signum function by sigmoid function can also reduce the chattering, but it will loss the robustness of SMO. More details can be found in [14]. However, in our work thanks to the obtained observer normal form (30), we reach a good estimation using only the high gain observer (HGO). The figures Fig.3 - Fig.4 show that for PMSM in d-q reference frame, the both methods (HGO, SMO) estimate successfully the states. Also, we note a less pronounced variation of estimation with the our method.

The load torque impact is noticeable on the figure Fig. 3 across the appearance of-overshoot when it changes the value. However the quality of the estimation is not affected. Therefore, we deduce that this observer is robust to external disturbance. Moreover, despite the difference of initial conditions between the simulation and estimation, it can be seen that the rotor shaft position value is correctly estimated. In other words, whatever the initial position of the rotor, the states of PMSM can be estimated. 

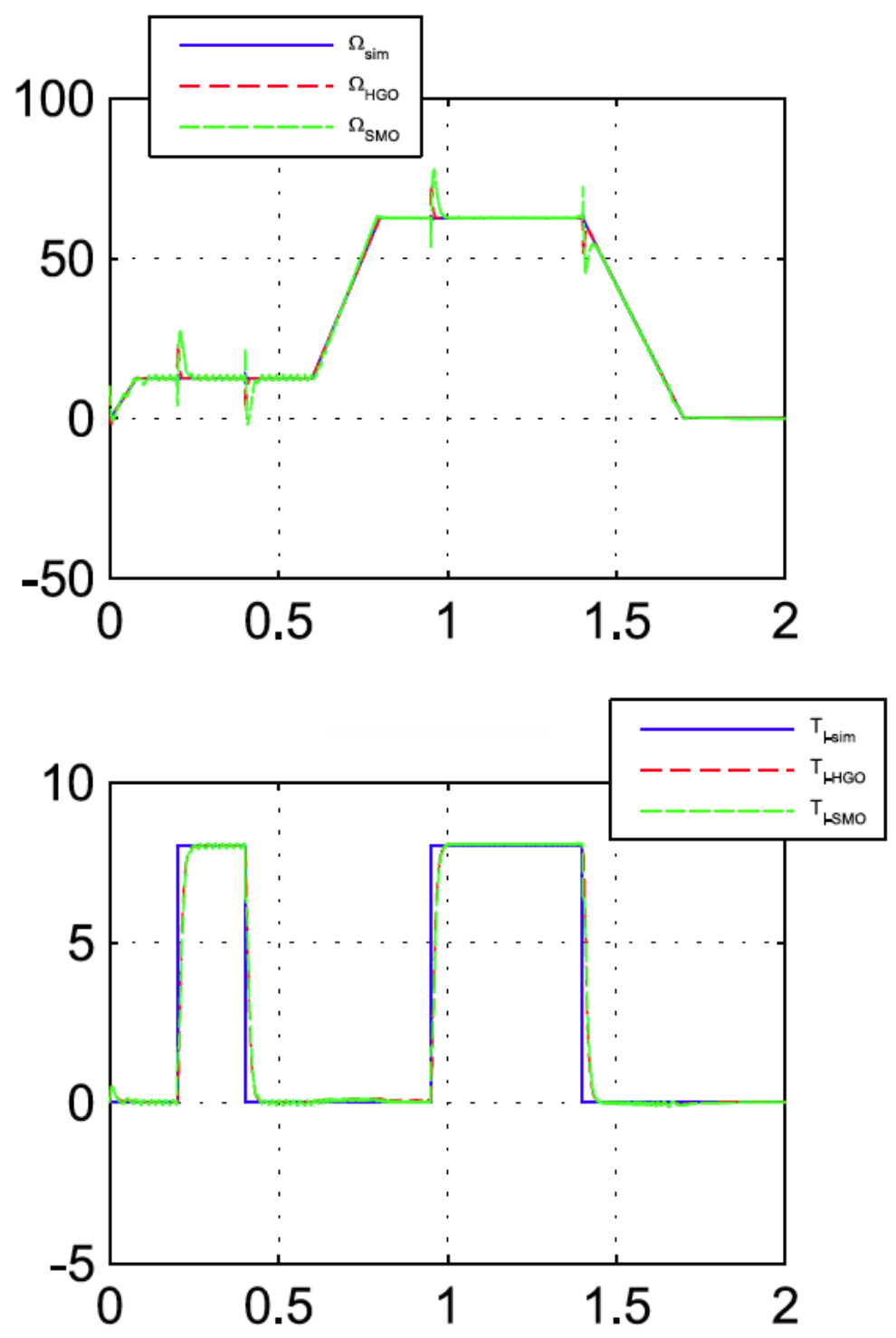

Fig. 3: Estimation of the rotor mechanical speed $\Omega$ and the load torque $T_{l}$. 


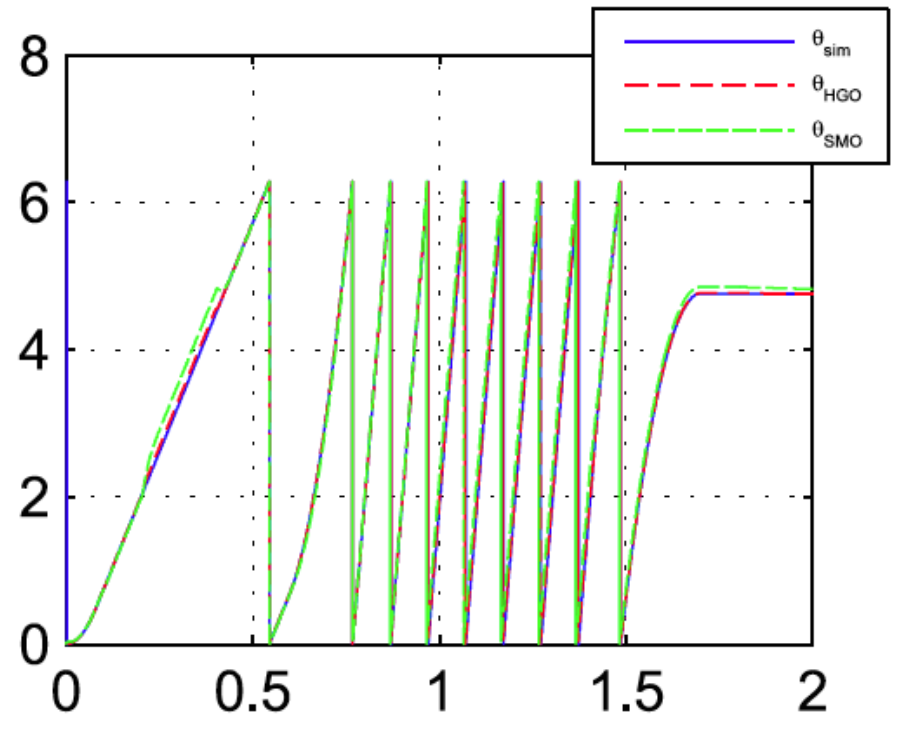

Fig. 4: Estimation of the rotor position $\theta$.

\subsection{Simulation results with adaptive observer}

To simultaneously estimate the states and the parameters of PMSM, we consider the dynamical form (32) and the adaptive observer (34)-(35) with $\Sigma=0.05 I_{d}, \rho_{z}=77$ an $\rho_{\delta}=60$. The performances of this method are show in Fig.5 - Fig.8.

Fig.5 - Fig. 6 show a good agreement in starting up between the estimated and the real values despite the uncertainty of the parameters values and the difference of initial conditions. We also note in Fig. 5 that the estimated speed follows their references. Moreover, the results of parameter estimation are shown in Fig.7 and Fig.8. The obtained result proves that the extended adaptive observer form has a very satisfactory performance. The obtained result demonstrates that the proposed approach can be considered as a suitable alternative to other methods, particularly when the simultaneously stimulation of states and parameters is required.

However, as expected, the rapid transients of load torque (disturbance) affect momentarily the estimation process. This phenomena is depicted through the appearance of-overshoot on the figures. In other words, a good converging performance of the estimated states and parameters can be observed, despite the appearance of overshoots due to the variation of load torque. It is worth noting that those over-shoots in estimation need to be carefully treated when designing observer-based controller. More precisely, if the designed controller depends on those estimations with over-shoot, the saturation function should be applied to mitigate the harmful influence of closed-loop system. This saturation can be normally realized since the states of system are always bounded in practice. 

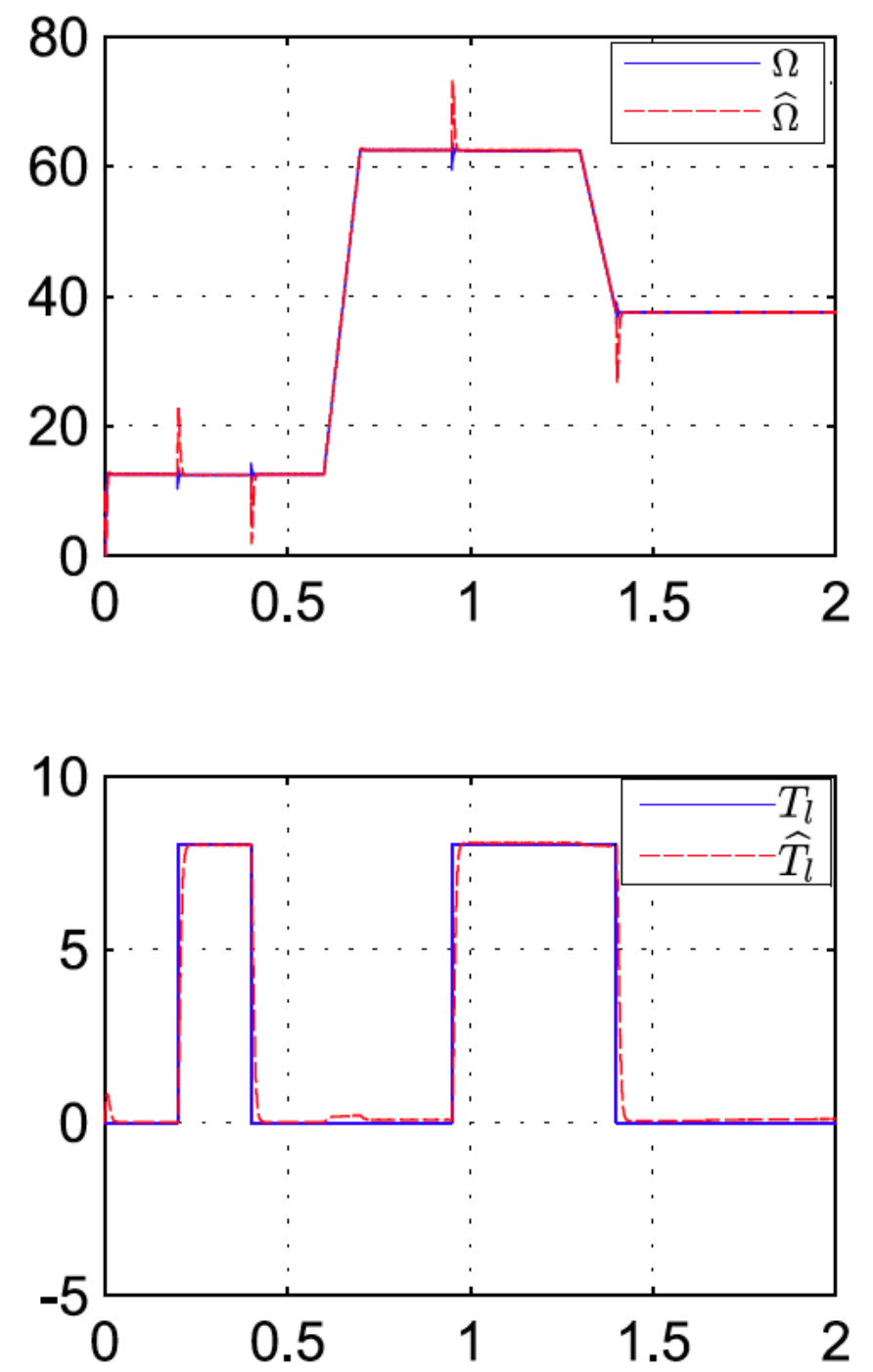

Fig. 5: Estimation of the rotor mechanical speed the load torque $T_{l}$. 


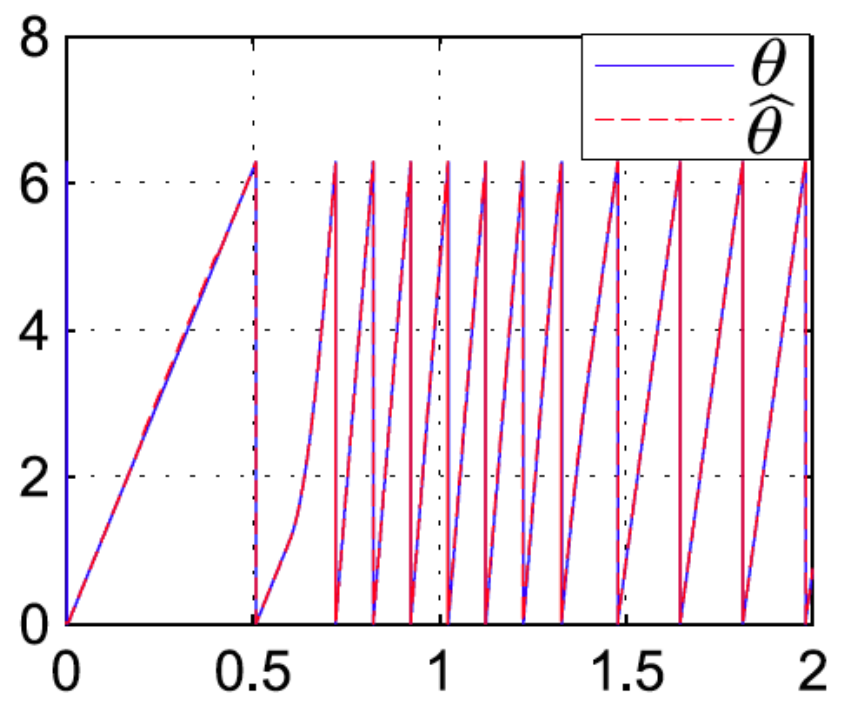

Fig. 6: Estimation of the rotor position $\theta$.

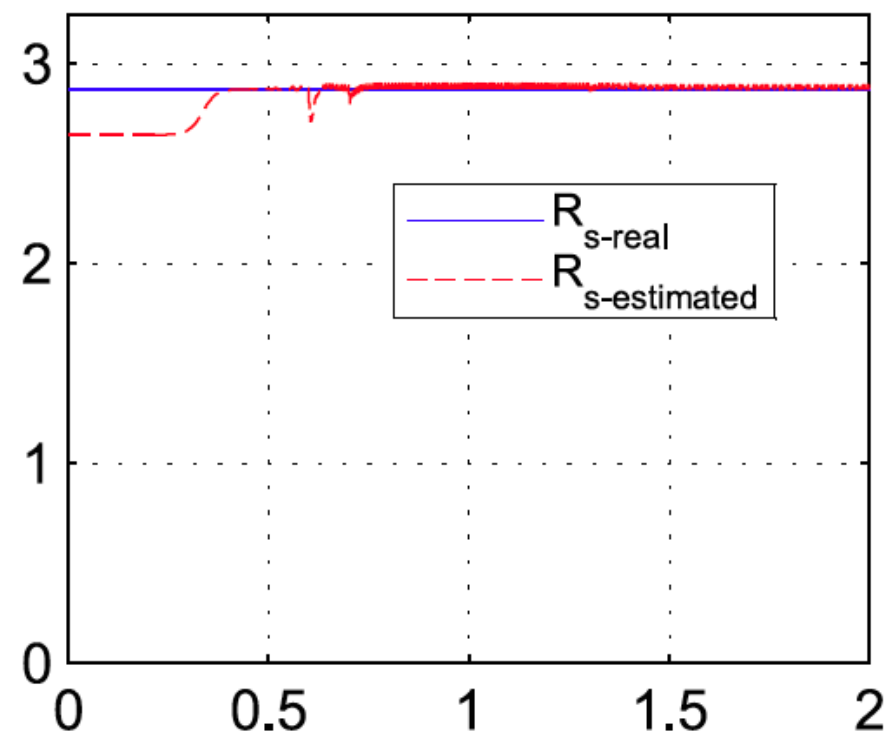

Fig. 7: Estimation of the stator resistor. 

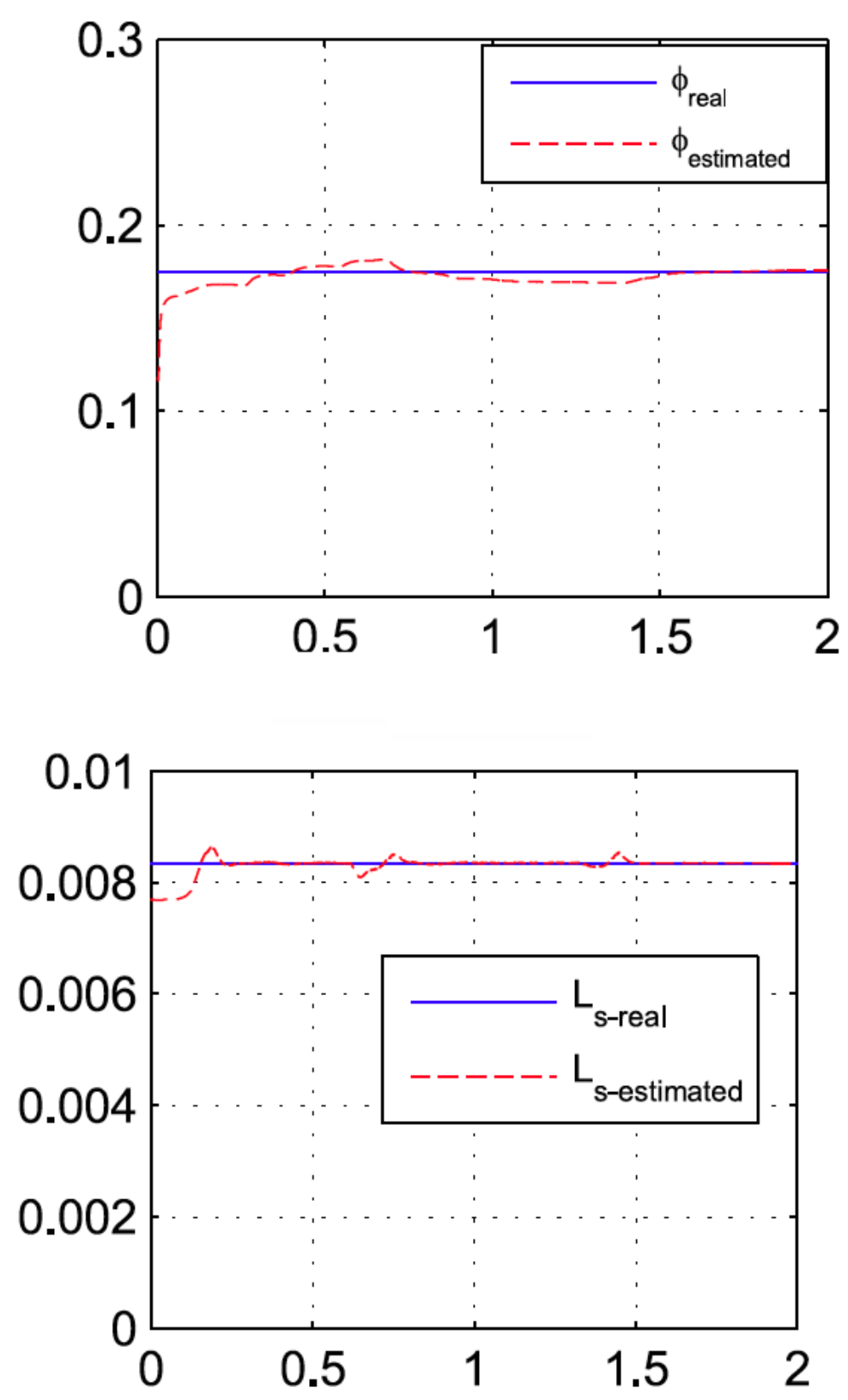

Fig. 8: Estimation of the magnet flux linkage and the stator inductance. 


\section{Conclusions}

This work proposes a sensorless PMSM control based on the concept of extended nonlinear observer normal forms. This enables us to apply a high gain observer or an adaptive observer to estimate the state and the parameters of PMSM. Compared to other methods (back-emf and MRAS methods) where the parameters should be well known, the extended adaptive observer approach, is easier to be implemented, and shows good dynamic performances in all speed ranges. Furthermore, the simultaneous estimation of states and parameters can have a big interest in the faults diagnosis and fault tolerant control based on auto-tuning and real time adaptation. Several simulation results have been presented to show the effectiveness of the proposed approach.

\section{Acknowledgments:}

The authors are deeply grateful to the reviewers for their thorough review and their interesting comments and suggestions that significantly contributed to improving the scientific content and the writing of this work.

\section{References}

[1] H. A. A. Awan, T. Tuovinen, S. E. Saarakkala, and M. Hinkkanen. Discrete-time observer design for sensorless synchronous motor drives. IEEE Transactions on Industry Applications, 52(5):3968-3979, 2016.

[2] J. Back, K. T. Yu, and J. H. Seo. Dynamic observer error linearization. Automatica, 42(12):2195-2200, 2006.

[3] G. Besançon, J. De Leon-Morales, and O. Huerta-Guevara. On adaptive observers for state affine systems. International journal of Control, 79(06):581-591, 2006.

[4] S. Bolognani, , R. Oboe, and M. Zigliotto. Sensorless full-digital pmsm drive with ekf estimation of speed and rotor position. IEEE Transactions on Industrial Electronics, 46(1):184-191, 1999.

[5] S. Bououden, D. Boutat, G. Zheng, J-P Barbot, and F. Kratz. A triangular canonical form for a class of 0-flat nonlinear systems. International Journal of Control, 84(2):261-269, 2011.

[6] M Boussak. Implementation and experimental investigation of sensorless speed control with initial rotor position estimation for interior permanent magnet synchronous motor drive. IEEE Transactions on Power Electronics, 20(6):1413-1422, 2005.

[7] D. Boutat. Geometrical conditions for observer error linearization via $\int 0,1, \ldots,(n-2)$. In 7th IFAC Symposium on Nonlinear Control Systems Nolcos'07, 2007.

[8] D. Boutat, A. Benali, H. Hammouri, and K. Busawon. New algorithm for observer error linearization with a diffeomorphism on the outputs. Automatica, 45(10):2187-2193, 2009. 
[9] D. Boutat and K. Busawon. On the transformation of nonlinear dynamical systems into the extended nonlinear observable canonical form. International Journal of Control, 84(1):94-106, 2011.

[10] D. Boutat, G. Zheng, J-P Barbot, and H. Hammouri. Observer error linearization multioutput depending. In Decision and Control, 2006 45th IEEE Conference on, pages 53945399. IEEE, 2006.

[11] K. Busawon, M. Farza, and H. Hammouri. A simple observer for a class of nonlinear systems. Applied Mathematics Letters, 11(3):27-31, 1998.

[12] J. Chiasson. Modeling and High-performance Control of Electric Machines. New York: John Wiley \& Sons, 2005.

[13] J.N. Chiasson and R.T. Novotnak. Nonlinear speed observer for the pm stepper motor. IEEE Transactions on Automatic Control, 38(10):1584-1588, 1993.

[14] C. Edwards and S. Spurgeon. Sliding Mode Control: Theory And Applications. Series in Systems and Control. Taylor \& Francis, 1998.

[15] M. Ezzat, J. de Leon, and A. Glumineau. Sensorless speed control of pmsm via adaptive interconnected observer. International Journal of Control, 84(11):1926-1943, 2011.

[16] M. Farza, I. Bouraoui, T. Ménard, R.-B. Abdennour, and M. MSaad. Adaptive observers for a class of uniformly observable systems with nonlinear parametrization and sampled outputs. Automatica, 50(11):2951-2960, 2014.

[17] M. Gan and C. Wang. An adaptive nonlinear extended state observer for the sensorless speed control of a pmsm. Mathematical Problems in Engineering, 2015.

[18] M. Ghanes and G. Zheng. On sensorless induction motor drives: sliding-mode observer and output feedback controller. IEEE Transactions on Industrial Electronics, 56(9):34043413, 2009.

[19] M.A. Hamida, J. De Leon, A. Glumineau, and R. Boisliveau. An adaptive interconnected observer for sensorless control of pm synchronous motors with online parameter identification. IEEE Transactions on Industrial Electronics, 60(2):739-748, 2013.

[20] M. Hou and A.C. Pugh. Observer with linear error dynamics for nonlinear multi-output systems. Systems \& Control Letters, 37:1-9, 1999.

[21] D. Janiszewski. Extended kalman filter based speed sensorless pmsm control with load reconstruction. In IEEE Conference on Industrial Electronics, pages 1465-1468, 2006.

[22] P. Jouan. Immersion of nonlinear systems into linear systems modulo output injection. SIAM Journal on Control and Optimisation, 41(6):1756-1778, 2003.

[23] H. Keller. Nonlinear observer design by transformation into a generalized observer canonical form. International Journal of Control, 46(6):1915-1930, 1987. 
[24] Ali J Koshkouei and Alan SI Zinober. Sliding mode state observation for non-linear systems. International Journal of Control, 77(2):118-127, 2004.

[25] A.J. Krener and A. Isidori. Linearization by output injection and nonlinear observers. Systems \& Control Letters, 3(1):47-52, 1983.

[26] A.J. Krener and W. Respondek. Nonlinear observers with linearizable error dynamics. SIAM Journal on Control and Optimization, 23(2):197-216, 1985.

[27] H. Lee and J. Lee. Design of iterative sliding mode observer for sensorless pmsm control. IEEE Transactions on Control Systems Technology, 21(4):1394-1399, 2013.

[28] W. Leonhard. Control of electrical drives. Springer, 2003.

[29] Y. Liang and Y. Li. Sensorless control of pm synchronous motors based on mras method and initial position estimation. In Sixth International Conference on Electrical Machines and Systems, ICEMS 2003, volume 1, pages 96-99. IEEE, 2003.

[30] M.V. Lopez, F. Plestan, and A. Glumineau. Linearization by completely generalized input ouput injection. Kybernetika, 35:793-802, 1999.

[31] A.F. Lynch and S.A. Bortoff. Nonlinear observers with approximately linear error dynamics: the multivariable case. IEEE Transactions on Automatic Control, 46(6):927-932, jun 2001.

[32] R. Marino and P. Tomei. Global adaptive observers for nonlinear systems via filtered transformations. IEEE Transactions on Automatic Control, 37(8):1239-1245, 1992.

[33] R. Marino and P. Tomei. Nonlinear control design: geometric, adaptive and robust. 1996.

[34] M. Mihoub, A. S. Nouri, and R. Ben Abdennour. A chattering free second order discrete sliding mode observer: An experimentation on a chemical reactor. In Control Automation (MED), 2010 18th Mediterranean Conference on, pages 739-744, 2010.

[35] B. Nahid-Mobarakeh, F. Meibody-Tabar, and F.-M Sargos. Mechanical sensorless control of pmsm with online estimation of stator resistance. IEEE Transactions on Industry Applications, 40(2):457-471, 2004.

[36] D. Noh, N.H. Jo, and J.H. Seo. Nonlinear observer design by dynamic observer error linearization. IEEE Transactions on Automatic Control, 49(10):1746-1753, 2004.

[37] S. Ogasawara and H. Akagi. An approach to position sensorless drive for brushless dc motors. IEEE Transactions on Industry Application, 27(5):928-933, 1991.

[38] V. Petrovic, R. Ortega, and A Stankovic. Interconnection and damping assignment approach to control of pm synchronous motors. Control Systems Technology, IEEE Transactions on, 9(6):811-820, Nov 2001.

[39] A. Phelps. On constructing nonlinear observers. SIAM Journal on Control and Optimmisation, 29:516-534, 1991. 
[40] Z. Qiao, T. Shi, Y. Wang, Y. Yan, C. Xia, and X. He. New sliding-mode observer for position sensorless control of permanent-magnet synchronous motor. IEEE Transactions on Industrial Electronics, 60(2):710-719, 2013.

[41] M Rashed and AF Stronach. A stable back-emf mras-based sensorless low-speed induction motor drive insensitive to stator resistance variation. IEE Proceedings-Electric Power Applications, 151(6):685-693, 2004.

[42] W. Respondek, A. Pogromsky, and H. Nijmeijer. Time scaling for observer design with linearizable error dynamics. Automatica, 40 (2):277-285, 2004.

[43] J. Rudolph and M. Zeitz. A block triangular nonlinear observer normal form. Systems \& Control Letters, 23(1):1-8, 1994.

[44] R. Tami, D. Boutat, and G. Zheng. Extended output depending normal form. Automatica, 49(7):2192 - 2198, 2013.

[45] R. Tami, D. Boutat, and G. Zheng. Nonlinear observer for the pm synchronous motor. In American Control Conference (ACC), pages 4133-4138. IEEE, 2014.

[46] D. Traoré, F. Plestan, A. Glumineau, and J. de Leon. Sensorless induction motor: highorder sliding-mode controller and adaptive interconnected observer. IEEE Transactions on Industrial Electronics, 55(11):3818-3827, 2008.

[47] Y. Wang and A. Lynch. Observer design using a generalized time-scaled block triangular observer form. Systems \& Control Letters, 58:346-352, 2009.

[48] X.H. Xia and W.B. Gao. Nonlinear observer with linearizable error dynamics. SIAM Journalon Control and Optimization, 27:199-216, 1989.

[49] Q. Zhang. Adaptive observer for multiple-input-multiple-output (mimo) linear timevarying systems. IEEE Transactions on Automatic Control, 47(3):525-529, 2002.

[50] G. Zheng and D. Boutat. Synchronisation of chaotic systems via reduced observers. IET Control Theory and Applications, 5(2):308, 2011.

[51] G. Zheng, D. Boutat, and J-P Barbot. Output dependent observability linear normal form. In Decision and Control, 2005 and 2005 European Control Conference. CDC-ECC'05. 44th IEEE Conference on, pages 7026-7030. IEEE, 2005.

[52] G. Zheng, D. Boutat, and J-P. Barbot. A single output dependent observability normal form. SIAM Journal on Control and Optimization, 46(6):2242-2255, 2007.

[53] G. Zheng, D. Boutat, and J-P Barbot. Multi-output dependent observability normal form. Nonlinear Analysis: Theory, Methods \& Applications, 70(1):404-418, 2009.

[54] Z. Zheng, Y. Li, and M. Fadel. Sensorless control of pmsm based on extended kalman filter. In Power Electronics and Applications, 2007 European Conference on, pages 1-8. IEEE, 2007. 
[55] G. Zhu, L.-A. Dessaint, O. Akhrif, and A. Kaddouri. Speed tracking control of a permanent-magnet synchronous motor with state and load torque observer. Industrial Electronics, IEEE Transactions on, 47(2):346-355, Apr 2000.

[56] G. Zhu, A. Kaddouri, L.-A. Dessaint, and O. Akhrif. A nonlinear state observer for the sensorless control of a permanent-magnet ac machine. IEEE Transactions on Industrial Electronics, 48(6):1098-1108, 2001. 